\title{
ASSOCIATION OF THE ACE rs4646994 AND rs4341 POLYMORPHISMS WITH THE PROGRESSION OF CAROTID ATHEROSCLEROSIS IN SLOVENIAN PATIENTS WITH TYPE 2 DIABETES MELLITUS
}

\author{
Merlo $\mathrm{S}^{1}$, Novák J $\mathrm{J}^{2,3,4}$, Tkáčová $\mathrm{N}^{2}$, Nikolajević Starčević $\mathrm{J}^{5}$, Šantl Letonja $\mathrm{M}^{6}$, Makuc J7, \\ Cokan Vujkovac A ${ }^{7}$, Letonja $\mathrm{J}^{5}$, Bregar $\mathrm{D}^{5}$, Zorc $\mathrm{M}^{5}$, Rojko $\mathrm{M}^{5}$, Mankoč $\mathrm{S}^{5}$, Kruzliak $\mathrm{P}^{8}$, Petrovič $\mathrm{D}^{5}$
}

*Corresponding Author: Professor Daniel Petrovič, M.D., Ph.D., Institute of Histology and Embryology, Faculty of Medicine University Ljubljana, Korytkova 2, SI-1000 Ljubljana, Slovenia. Tel: +386-1-543-7367. Fax: +386-1543-7361.E-mail: daniel.petrovic@mf.uni-lj.si

\begin{abstract}
The current study was designed to reveal possible associations between the angiotensin-converting-enzyme ( $A C E$ ) gene polymorphisms (rs4646994 and rs4341) with markers of carotid atherosclerosis in patients with type 2 diabetes mellitus (T2DM) in a 4-year-long follow-up study. Five hundred and ninety-five T2DM subjects and 200 control subjects were enrolled. Genotyping of ACE polymorphisms was performed using KASPar assays, and ultrasound examinations were performed twice (at the enrollment and at follow-up). With regard to the progression of atherosclerosis in subjects with T2DM, statistically significant differences were demonstrated in the change of the sum of carotid plaques thickness for the
\end{abstract}

\footnotetext{
${ }^{1}$ Institute of Oncology Ljubljana, Zaloška 2, 1000 Ljubljana, Slovenia

2 Department of Physiology, Faculty of Medicine, Masaryk University, Kamenice 5, building A20, 625 00, Brno, Czech Republic

${ }^{3}$ Department of Pathological Physiology, Faculty of Medicine, Masaryk University, Kamenice 5, building A18, 62500 Brno, Czech Republic

${ }^{4}$ Second Department of Internal Medicine, St. Anne's University Hospital and Faculty of Medicine, Masaryk University, Brno, Czech Republic

${ }^{5}$ Institute of Histology and Embryology, Faculty of Medicine, University in Ljubljana, Vrazov trg 2, Ljubljana, Slovenia

${ }^{6}$ General Hospital Rakičan, Ulica dr. Vrbnjaka 6, Murska Sobota, 9000, Slovenia

${ }^{7}$ General Hospital Slovenj Gradec, Gosposvetska cesta 1, 2380 Slovenj Gradec, Slovenia

${ }^{8}$ Department of Cardiovascular Diseases, International Clinical Research Center, St. Anne's University Hospital and Masaryk University, Brno, Czech Republic
}

rs4646994 polymorphism. We did not demonstrate an association between the tested polymorphisms (rs4646994 and rs4341) and either carotid intima media thickness (CIMT) or CIMT progression in a 3.8-year period. In our study, we demonstrated that subjects with T2DM with the DD genotype of the rs4646994 [ACE insertion/deletion (I/D)] polymorphism had faster progression of atherosclerosis in comparison to subjects with other genotypes.

Keywords: Angiotensin-converting-enzyme $(A C E)$ gene polymorphism; Association study; Carotid atherosclerosis; Type 2 diabetes mellitus (T2DM).

\section{INTRODUCTION}

Type 2 diabetes mellitus (T2DM) represents a chronic illness characterized by the disability of the body to utilize glucose either because of insulin resistance in peripheral tissues or because of a decreased production of insulin by the pancreas [1]. Type 2 diabetes mellitus is known to promote the atherosclerotic process, which is characterized by endothelial dysfunction and by accumulation of foam cells and vessel wall inflammation. As the process continues, the narrowing of the vessel lumen occurs, leading to acute cardiovascular events [2].

The renin-angiotensin-aldosterone system is one of the main regulators of blood pressure having also other local (tissue-specific) roles [3]. Genetic polymorphisms in different parts of this system have previously been described to associate with various cardiovascular and other diseases, with the angio- 
tensin-converting-enzyme (ACE) insertion/deletion (I/D) polymorphism representing one of the most commonly studied polymorphisms that affects circulating ACE levels [3]. This polymorphism has recently been shown to be in linkage disequilibrium with another ACE polymorphism, rs4341 [3]; however, data about these two polymorphism and their possible association with carotid atherosclerosis in patients with diabetes mellitus are limited.

The present study was thus designed to investigate the association between polymorphisms of the $A C E$ gene (rs 4646994 and rs4341) and markers of carotid atherosclerosis [carotid intima media thickness (CIMT), number of affected segments of carotid arteries and sum of plaques thickness] in patients with T2DM. The second aim was to see whether these two polymorphisms (rs4646994 and rs4341) affect progression of carotid atherosclerosis in a 4-year follow-up.

\section{MATERIALS AND METHODS}

In this cross-sectional study, 595 (338 males; 257 females) subjects with T2DM and 200 (92 males; 108 females) subjects without T2DM (control group) were enrolled as described previously [4]. The study protocol was approved by the Slovene Medical Ethics Committee (128/09/ 2010). After informed consent for participation in the study was obtained, a detailed interview was made.

All ultrasound examinations were performed by two experienced doctors blinded to the participants' diabetes status. The CIMT, defined as the distance from the leading edge of the lumen-intima interface to the leading edge of the media-adventitia interface, was measured as described previously [4]. Plaques were defined as a focal intima-media thickening and divided according to their echogenic/ echolucent characteristics into five types as described previously [4]. The inter-observer reliability for carotid plaque characterization was found to be substantial $(\kappa=$ $0.64, p<0.001)$.

Blood samples for biochemical analyses were collected as described previously [4]. The genomic DNA was extracted from $100 \mu \mathrm{L}$ of whole blood using a FlexiGene DNA isolation kit, in accordance with the recommended protocol (Qiagen $\mathrm{GmbH}$, Hilden, Germany). The ACE polymorphisms (rs4646994 and rs4341) were determined by a novel fluorescence- based competitive allele-specific polymerase chain reaction (PCR) (KASPar; Kbioscience Ltd., Hoddesdon, Hertfordshire, UK), assay. Details of the method used can be found at http://www.kbioscience. co.uk/.

Continuous variables were expressed as means \pm standard deviations (SDs) if normally distributed, and as median (interquartile range) if asymmetrically distributed. Continuous clinical data were compared using an unpaired Student's $t$-test or analysis of variance (ANOVA) when normally distributed and the Mann-Whitney U test or the Kruskal-Wallis H test when asymmetrically distributed. The Pearson $\chi^{2}$ test was used to compare discrete variables.

To determine the association of the $A C E$ gene polymorphisms (rs4646994 and rs4341) with CIMT, a multiple linear regression analysis was performed. We used an additive model in which common allele homozygotes were coded as 1 , heterozygotes as 2 , and rare allele homozygotes as 3 . All the regression models were adjusted for the presence of well-established cardiovascular risk factors. The results are presented as standardized $\beta$ coefficients and $p$ values for the linear regression and by odds ratios (ORs) and $95 \%$ confidence intervals (CIs) for the logistic regression. A two-tailed $p$ value of less than 0.05 was considered statistically significant. A statistical analysis was performed using the Statistical Package for the Social Science (SPSS) software for Windows, version 20 (SPSS Inc., Chicago, IL, USA).

\section{RESULTS}

Patients with T2DM had a greater waist circumference, higher fasting glucose and $\mathrm{Hb} \mathrm{A}_{1 \mathrm{c}}$ levels compared to controls, whereas there were no statistically significant differences in age, body mass index (BMI), and systolic and diastolic blood pressure between patients with T2DM and control subjects (Table 1). Patients with T2DM had lower total, high density lipoprotein (HDL) and low density lipoprotein (LDL) cholesterol levels and higher triglyceride levels compared to controls (Table 1). Plasma levels of inflammatory markers (i.e., high sensitive C-reactive protein (hs-CRP) were statistically significantly higher in patients with T2DM compared to controls (Table 1).

The genotype distributions both in patients with T2DM and controls were in the Hardy-Weinberg equilibrium for both $A C E$ gene polymorphisms 
Table 1. Baseline characteristics of subjects with type 2 diabetes mellitus and control subjects without type 2 diabetes mellitus.

\begin{tabular}{|c|c|c|c|}
\hline Parameters & T2DM Subjects $(n=595)$ & Control Subjects $(n=200)$ & $p$ Value \\
\hline Age (years) & $61.38 \pm 9.65$ & $60.07 \pm 9.18$ & 0.07 \\
\hline $\begin{array}{c}\text { Gender: } \\
\text { males } \\
\text { females }\end{array}$ & $\begin{array}{l}338(56.8 \%) \\
257(43.2 \%)\end{array}$ & $\begin{array}{r}92(46.0 \%) \\
108(54.0 \%)\end{array}$ & 0.008 \\
\hline Duration of T2DM (years) & $11.25 \pm 7.88$ & - & - \\
\hline Cigarette smokers (\%) & $53(8.9 \%)$ & $34(17.0 \%)$ & 0.002 \\
\hline Waist circumference $(\mathrm{cm})$ & $108.65 \pm 12.88$ & $93.31 \pm 13.18$ & $<0.001$ \\
\hline BMI $\left(\mathrm{kg} / \mathrm{m}^{2}\right)$ & $30.96 \pm 4.74$ & $27.90 \pm 4.42$ & 0.16 \\
\hline $\mathrm{SBP}(\mathrm{mmHg})$ & $146.98 \pm 19.98$ & $143.30 \pm 16.6$ & 0.86 \\
\hline $\mathrm{DBP}(\mathrm{mmHg})$ & $85.75 \pm 11.62$ & $84.70 \pm 11.6$ & 0.19 \\
\hline Fasting glucose $(\mathrm{mmol} / \mathrm{L})$ & $8.04 \pm 2.57$ & $5.27 \pm 0.87$ & $<0.001$ \\
\hline $\mathrm{Hb} \mathrm{A}_{1 \mathrm{c}}(\%)$ & $7.89 \pm 3.56$ & $4.79 \pm 0.29$ & $<0.001$ \\
\hline Total cholesterol (mmol/L) & $4.70 \pm 1.19$ & $5.36 \pm 1.08$ & $<0.001$ \\
\hline HDL cholesterol (mmol/L) & $1.19 \pm 0.35$ & $1.43 \pm 0.37$ & $<0.001$ \\
\hline LDL cholesterol (mmol/L) & $2.63 \pm 0.94$ & $3.24 \pm 0.98$ & $<0.001$ \\
\hline Triglycerides $(\mathrm{mmol} / \mathrm{L})$ & $1.9(1.2-2.7)$ & $1.3(0.9-1.9)$ & $<0.001$ \\
\hline hs-CRP (mg/L) & $2.2(1.0-4.3)$ & $1.3(0.8-2.7)$ & $<0.001$ \\
\hline CIMT $(\mu \mathrm{m})$ & $1013.0 \pm 208.0$ & $979.0 \pm 141.0$ & 0.03 \\
\hline
\end{tabular}

Continuous variables are expressed as means \pm SDs if normally distributed and as median (interquartile range) if asymmetrically distributed. Categorical variables are expressed as frequency (percentage). BMI: body mass index; SBP: systolic blood pressure; DBP: diastolic blood pressure; Hb $\mathrm{A}_{1 \mathrm{c}}$ : glycated hemoglobin (Hb); HDL: high density lipoprotein; LDL: low density lipoprotein; hs-CRP: high sensitivity C-reactive protein; CIMT: carotid intima media thickness.

[rs4646994: T2DM (genotype frequencies: II genotype $21.5 \%$, ID genotype $46.4 \%$, DD genotype $32.1 \%$; $\chi^{2}=2.27 ; p=0.13$ ) and controls (genotype frequencies: II genotype $24.0 \%$, ID genotype $49.0 \%$, DD genotype $\left.27.0 \% ; \chi^{2}=0.07 ; p=0.78\right)$; rs 4341 : T2DM (genotype frequencies: GG genotype $28.1 \%$, GC genotype $52.1 \%$, CC genotype $19.8 \% ; \chi^{2}=1.44 ; p=0.23$ ) and controls (genotype frequencies: GG genotype $23.5 \%$, GC genotype $54.5 \%$, CC genotype $22.0 \%$; $\left.\left.\chi^{2}=1.63 ; p=0.20\right)\right]$. No statistically significant differences in the ACE rs4646994 and rs4341 genotype distribution frequencies were observed between the T2DM patients and controls. Moreover, in our study, linkage disequilibrium between the two selected single nucleotide polymorphisms (SNPs) (rs4646994 and rs4341) was confirmed (d' $\left.=0.98 r^{2}=0.82\right)$.

Several parameters of carotid atherosclerosis, such as CIMT, number of involved segments, and sum of plaque thicknesses, were evaluated with regard to different genotypes of both ACE polymorphisms in subjects with T2DM at enrollment and after 3.8 years (Table 2). Moreover, the parameters of progression of atherosclerosis, i.e., annual increase in CIMT, change in number of segments with plaques and change in the sum of carotid plaque thicknesses, were analyzed with univariate and multiple linear regression analyses (Tables 3 and 4). With regard to the progression of atherosclerosis in subjects with T2DM, statistically significant differences were demonstrated in the change of the sum of carotid plaque thickness for the rs4646994 polymorphism only (Table 3). Finally, according to the results of multiple linear regression analysis, faster progression of atherosclerosis was demonstrated in subjects with T2DM with the DD genotype of the rs 4646994 (ACE I/D) polymorphism in comparison with subjects with other genotypes (Table 4).

\section{DISCUSSION}

In this study, we demonstrated the effect of the DD genotype of the rs4646994 (ACE I/D) polymorphism on atherosclerosis progression in subjects with T2DM. Statistically significant differences in a 3.8year observation period were found in the change in the sum of carotid plaques for the rs4646994 polymorphism. Our findings are in accordance with the findings of Saitou et al. [5], who on a cohort of 222 
Table 2. Comparison of markers of carotid atherosclerosis in subjects with type 2 diabetes mellitus at the beginning and the end of the study with regard to the rs4646994 (angiotensin-converting-enzyme insertion/deletion) and rs4341 polymorphisms.

\begin{tabular}{|l|c|c|c|c|c|c|c|c|}
\hline $\begin{array}{l}\text { rs4646994 } \\
\text { (ACE I/D) }\end{array}$ & \multicolumn{5}{|c|}{ Enrollment } & \multicolumn{4}{c|}{ Endpoint } \\
\hline & II & ID & DD & $p$ Value & II & ID & DD & $p$ Value \\
\hline $\begin{array}{l}\text { Intima media } \\
\text { thickness }(\mu \mathrm{m})\end{array}$ & $998.0 \pm 147.0$ & $1002.0 \pm 178.0$ & $1012.0 \pm 178.0$ & 0.62 & $1048.0 \pm 147.0$ & $1054.0 \pm 128.0$ & $1060.0 \pm 164.0$ & 0.59 \\
\hline $\begin{array}{l}\text { Involved } \\
\text { segments }(n)\end{array}$ & $2.65 \pm 1.49$ & $3.04 \pm 1.71$ & $2.66 \pm 1.44$ & 0.12 & $4.48 \pm 1.75$ & $4.62 \pm 1.68$ & $4.71 \pm 1.76$ & 0.68 \\
\hline $\begin{array}{l}\text { Sum of plaque } \\
\text { thickness }(\mathrm{mm})\end{array}$ & $7.92 \pm 3.47$ & $8.99 \pm 3.38$ & $7.67 \pm 3.14$ & 0.09 & $10.17 \pm 4.29$ & $10.08 \pm 5.26$ & $11.38 \pm 5.32$ & 0.76 \\
\hline
\end{tabular}

\begin{tabular}{|l|c|c|c|c|c|c|c|c|}
\hline rs4341 & \multicolumn{4}{|c|}{ Enrollment } & \multicolumn{4}{c|}{ Endpoint } \\
\hline & GG & GC & CC & $p$ Value & GG & GC & CC & $p$ Value \\
\hline $\begin{array}{l}\text { Intima media } \\
\text { thickness }(\mu \mathrm{m})\end{array}$ & $1022.0 \pm 212.0$ & $1002.0 \pm 217.0$ & $1018.0 \pm 191.0$ & 0.71 & $1065.0 \pm 155.0$ & $1054.0 \pm 163.0$ & $1051.0 \pm 164.0$ & 0.62 \\
\hline $\begin{array}{l}\text { Involved } \\
\text { segments }(n)\end{array}$ & $2.49 \pm 1.48$ & $2.54 \pm 1.73$ & $2.44 \pm 1.57$ & 0.89 & $3.60 \pm 1.76$ & $3.70 \pm 1.64$ & $3.70 \pm 1.78$ & 0.96 \\
\hline $\begin{array}{l}\text { Sum of plaque } \\
\text { thickness }(\mathrm{mm})\end{array}$ & $7.77 \pm 4.50$ & $8.02 \pm 4.42$ & $7.53 \pm 4.77$ & 0.74 & $9.38 \pm 5.34$ & $9.16 \pm 5.16$ & $8.72 \pm 4.56$ & 0.88 \\
\hline
\end{tabular}

ACE I/D: angiotensin-converting-enzyme insertion/deletion.

Table 3. Changes of markers of carotid atherosclerosis in subjects with type 2 diabetes mellitus between first the examination and the examination at the end of the study with regard to the rs4646994 (angiotensin-converting-enzyme insertion/deletion) and rs4341 polymorphisms.

\begin{tabular}{|l|c|c|c|c|}
\hline rs4646994 ACE I/D & II & ID & DD & $p$ Value \\
\hline Annual increase in CIMT $(\mu \mathrm{m} /$ year $)$ & $14.28(5.35-26.83)$ & $22.43(16.73-32.19)$ & $20.34(10.53-33.65)$ & 0.34 \\
\hline$\Delta$ Number of segments with plaques & $3.0(1.0-3.0)$ & $1.0(0.5-2.5)$ & $2.0(1.0-3.0)$ & 0.42 \\
\hline$\Delta$ Sum of carotid plaques thickness $(\mathrm{mm})$ & $4.00(2.30-5.30)$ & $4.68(3.30-7.60)$ & $6.22(3.90-8.10)$ & 0.04 \\
\hline rs4341 & GG & GC & CC & $p$ Value \\
\hline Annual increase in CIMT $(\mu \mathrm{m} /$ year) & $21.05(14.28-33.65)$ & $20.69(16.55-24.26)$ & $14.28(10.71-20.08)$ & 0.26 \\
\hline$\Delta$ Number of segments with plaques & $2.0(1.0-3.5)$ & $2.0(1.0-2.5)$ & $3.0(2.0-3.0)$ & 0.49 \\
\hline$\Delta$ Sum of carotid plaques thickness $(\mathrm{mm})$ & $4.60(3.40-7.90)$ & $5.60(4.35-8.55)$ & $5.6(2.60-6.90)$ & 0.07 \\
\hline
\end{tabular}

ACE I/D: angiotensin-converting-enzyme insertion/deletion. Annual increase in CIMT (carotid intima media thickness) was calculated as CIMT(beginning)-CIMT(endpoint)/follow-up in years. Change in number of plaques is expressed as number of segments with plaque at the endpoint minus the number at the beginning. Sum of plaque thickness is calculated as the end sum minus the beginning sum. Data are expressed as median and range.

Table 4. Multiple linear regression analysis for association of rs4646994 (angiotensin-converting-enzyme insertion/deletion) with carotid atherosclerosis progression in patients with type 2 diabetes mellitus.

\begin{tabular}{|l|c|c|c|c|c|c|}
\hline Parameters & \multicolumn{2}{|c|}{$\Delta$ CIMT/Year } & \multicolumn{2}{c|}{$\Delta$ Number of Segments } & \multicolumn{2}{c|}{$\Delta$ Sum of Plaque Thickness } \\
\hline & $\beta$ & $p$ Value & $\beta$ & $p$ Value & $\beta$ & $p$ Value \\
\hline A) rs4646994 & & & & & & 0.272 \\
\hline Hypertension (yes/no) & 0.144 & 0.59 & 0.206 & 0.88 & 0.53 \\
\hline Systolic blood pressure & 0.031 & 0.42 & 0.027 & 0.15 & 0.014 & 0.69 \\
\hline ID & 0.141 & 0.49 & 0.116 & 0.51 & 0.845 & 0.26 \\
\hline DD & 0.102 & 0.63 & 0.146 & 0.41 & 0.952 & $\mathbf{0 . 0 4}$ \\
\hline
\end{tabular}

All models were adjusted to age, sex, smoking habits, serum levels of $\mathrm{Hb} \mathrm{A}_{1 \mathrm{c}}$, statin treatment and initial values of the dependent variables. Reference groups are homozygotes for the I allele. 
T2DM patients reported faster progression of atherosclerosis in subjects with the D allele.

Moreover, in our study, we did not demonstrate an association between tested polymorphisms (rs4646994, rs4341) and either CIMT or CIMT progression in an almost 4-year period. In 1994, it was reported for the first time that ACE levels correlate with CIMT in healthy persons [6]. As the ACE I/D polymorphism is known to affect circulating $\mathrm{ACE}$ levels (subjects with the DD genotype having the highest serum ACE levels compared to subjects with other genotypes) [7], subsequent studies have already been conducted to identify a possible association of the ACE I/D genotype with atherosclerosis, both on general and diabetic populations, providing contradictory results [5-10]. Using smaller or larger cohorts of the general population, association of the ACE I/D polymorphism with carotid atherosclerosis was confirmed in some studies [6-8] and opposed by others [9]. Finally, large meta-analyses confirmed statistically significant association of ACE I/D with CIMT in the general population [10].

In studies that did not focus on the general population but on patients with T2DM, Kogawa et al. [11] in 1997, studied femoral and CIMT in healthy persons and patients with T2DM, and showed that only in T2DM patients was there a significant association of the D allele with higher CIMT. Since the study of Kogawa et al. [11], studies focusing on the association of CIMT and the ACE I/D polymorphism provided contradictory results, similar to studies conducted in the general population. In the diabetic heart study no association was found between ACE I/D and CIMT [12], similar to the study focusing on the offspring of patients with T2DM [13]. On the other hand, Sticchi et al. [14] reported a higher risk of carotid stenosis in D allele carriers, and Zhou et al. [15] reported higher lipid levels in older patients with T2DM carrying the D allele, which could promote atherosclerosis progression.

Certain limitations of our study should be noted. First is the moderate sample size of our study. However, all the participants were enrolled from an ethnically homogenous population, which minimizes possible biases from population stratification. Second, the results of our study may be affected by statin therapy, and antihypertensive agents, and these facts were not appreciated in the statistical analysis.

In conclusion, our study represents the first larger study focusing on the effect of two ACE polymor- phisms (rs4646994 and rs4341) on the progression of carotid atherosclerosis in subjects with T2DM. We demonstrated that those subjects with T2DM with the DD genotype of the rs4646994 (ACE I/D) polymorphism had faster progression of atherosclerosis in comparison with subjects with other genotypes.

\section{ACKNOWLEDGMENTS}

The authors thank Mrs. Visam Bajt, BA, for revising the English.

Declaration of Interest. The authors report no conflicts of interest. The authors alone are responsible for the content and writing of this article.

\section{REFERENCES}

1. Grundy SM, Brewer HB Jr, Cleeman JI, Smith SC Jr, Lenfant C. Definition of metabolic syndrome: Report of the National Heart, Lung, and Blood Institute/American Heart Association conference on scientific issues related to definition. Arterioscler Thromb Vasc Biol. 2004; 24(2): e13-e18.

2. Hopkins PN. Molecular biology of atherosclerosis. Physiol Rev. 2013; 93(3): 1317-1542.

3. Castellon R, Hamdi HK. Demystifying the ACE polymorphism: From genetics to biology. Curr Pharm Des. 2007; 13(12): 1191-1198.

4. Nikolajevic Starcevic J, Santl Letonja M, Praznikar ZJ, Makuc J, Vujkovac AC, Petrovic D. Polymorphisms XbaI (rs693) and EcoRI (rs1042031) of the ApoB gene are associated with carotid plaques but not with carotid intima-media thickness in patients with diabetes mellitus type 2 . Vasa. 2014; 43(3): 171-180.

5. Saitou M, Osonoi T, Kawamori R, Katakami $\mathrm{N}$, Kaneto H, Matsuhisa M, et al. Genetic risk factors and the anti-atherosclerotic effect of pioglitazone on carotid atherosclerosis of subjects with type 2 diabetes - A retrospective study. J Atheroscler Thromb. 2010; 17(4): 386-394.

6. Bonithon-Kopp C, Ducimetiere P, Touboul PJ, Feve JM, Billaud E, Courbon D, et al. Plasma angiotensin-converting enzyme activity and carotid wall thickening. Circulation. 1994; 89(3): 952-954. 
7. He Q, Fan C, Yu M, Wallar G, Zhang ZF, Wang $\mathrm{L}$, et al. Associations of ACE gene insertion/ deletion polymorphism, ACE activity, and ACE mRNA expression with hypertension in a Chinese population. PLoS One. 2013; 8(10): e75870. doi: 10.1371/journal.pone.0075870.

8. Castellano M, Muiesan ML, Rizzoni D, Beschi M, Pasini G, Cinelli A, et al. Angiotensin-converting enzyme I/D polymorphism and arterial wall thickness in a general population. The Vobarno Study. Circulation. 1995; 91(11): 2721-2724.

9. Islam MS, Lehtimaki T, Juonala M, Kahonen M, Hutri-Kahonen N, Kainulainen K, et al. Polymorphism of the angiotensin-converting enzyme (ACE) and angiotesinogen (AGT) genes and their associations with blood pressure and carotid artery intima media thickness among healthy Finnish young adults - The Cardiovascular risk in young Finns study. Atherosclerosis. 2006; 188(2): 316-322.

10. Paternoster L, Martinez-Gonzalez NA, Charleton R, Chung M, Lewis S, Sudlow CL. Genetic effects on carotid intima-media thickness: Systematic assessment and meta-analyses of candidate gene polymorphisms studied in more than 5000 subjects. Circ Cardiovasc Genet. 2010; 3(1): 15-21.
11. Kogawa K, Nishizawa Y, Hosoi M, Kawagishi T, Maekawa K, Shoji T, et al. Effect of polymorphism of apolipoprotein $\mathrm{E}$ and angiotensinconverting enzyme genes on arterial wall thickness. Diabetes. 1997; 46(4): 682-687.

12. Burdon KP, Langefeld CD, Wagenknecht LE, Carr JJ, Freedman BI, Herrington D, et al. Association analysis of genes in the renin-angiotensin system with subclinical cardiovascular disease in families with type 2 diabetes mellitus: The diabetes heart study. Diabet Med. 2006; 23(3): 228-234.

13. Purnamasari D, Widjojo BD, Antono D, Syampurnawati M. ACE gene polymorphism and atherosclerotic lesion of carotid artery among offsprings of type 2 diabetes mellitus. Acta Med Indones. 2012; 44(2): 128-134.

14. Sticchi E, Romagnuolo I, Sofi F, Pratesi G, Pulli $\mathrm{R}$, Pratesi $\mathrm{C}$, et al. Association between polymorphisms of the renin angiotensin system and carotid stenosis. J Vasc Surg. 2011; 54(2): 467473.

15. Zhou YF, Yan H, Hou XP, Miao JL, Zhang J, Yin QX, et al. Association study of angiotensin converting enzyme gene polymorphism with elderly diabetic hypertension and lipids levels. Lipids Health Dis. 2013; 12(1): 187-191. 